\title{
Guest Editorial: Quantum Dots
}

\author{
Michael Gerhold ${ }^{\mathrm{a}}$ and Jian $\mathrm{Xu}^{\mathrm{b}}$ \\ ${ }^{a}$ US Army Research Office, Research Triangle Park, NC 27703 \\ mike.gerhold@us.army.mil \\ ${ }^{\mathrm{b}}$ Department of Engineering Science and Mechanics, Pennsylvania State University, \\ University Park, PA 16802 \\ jianxu@psu.edu
}

Advances in quantum dot (QD) waveguides, lasers, photodetectors, and growth/synthesis technologies are the subject of this special section. The development of Stranski-Krastanow strain-layer QDs has led to the emergence of low-threshold and moderate to high bandwidth semiconductor lasers in the 0.85 - to 1.5 - $\mathrm{mm}$ wavelength range mainly based on the InGaAs system. Advances such as lower threshold, improved temperature performance, and high modulation bandwidths have been reported. Advances in epitaxially grown QD uniformity and density led to laser companies such as QD Laser of Tokyo, a Joint Venture of Fujitsu and Mitsui Venture Capital Corp., spun-off from Arakawa's group at the University of Tokyo. Innolume is another company based on epitaxially grown QD technology for lasers and semiconductor optical amplifiers (SOAs) spun-off from research in the past 16 years.

In this special section comprising eight invited articles, important recent developments which may lead to further device improvements are in focus. Research on QD and nanowire lasers grown on silicon, methods of forming higher-density and higher-quality QDs, applications of QDs for mid-infrared lasers, and QD photodetectors in the mid-infrared regime are reported.

In the late 1990s, QD lasers were developed at $\sim 1-\mu \mathrm{m}$ wavelength. The push toward 1.3 $\mu \mathrm{m}$ and beyond saw drops in modulation bandwidth, effects which are still being studied. Of late though, QD lasers have been made on silicon out to $1.5-\mu \mathrm{m}$ wavelength, which approaches the window needed for minimum loss in optical fibers. Also, interfacing such lasers with Si CMOS has been a challenge. Zetian Mi, who has studied the problem of growing QD lasers on silicon and has made great strides in the production of such devices but also realizes their shortcomings, presented at 2009 SPIE Photonics West new ways to overcome the strain built up due to lattice mismatch. Other approaches and techniques summarized in a review article by Mi and Chang include nanowires of radial and axial orientations on silicon for various materials.

The second forefront area comprises novel methods of formation of QDs to improve uniformity and density, as discussed by Park et al. Several approaches are possible using nanopatterning techniques, but one of the most promising ones is the use of new chemistries based on di-block co-polymer matrices which form dense but uniform nanoscale patterns for highly uniform QDs - in this case — of InP, which are potentially better than InAs QDs due to reduced surface recombination velocities.

Another area of study in QD growth is that of droplet epitaxy. In the article by Mano et al., recent developments in the self-assembly of GaAs quantum nanostructures by droplet epitaxy are reviewed. Additionally, the authors describe their micro-photoluminescence experiments to clarify the electronic states and relaxation processes in the nanostructures. Details are given to unveil the lasing effect, excitonic Rabi oscillations, and the Purcell effect in GaAs quantum dots.

Theoretical investigations of QD lasers are also still progressing, particularly by Levon Asryan's group. Multiple parameters such as size, uniformity, and number of layers are being 
varied to optimize laser designs and plan for future improvements based on experimental advances. The effects of multi-longitudinal mode behavior, internal loss, and multiple transitions are being elucidated. Asryan reviews the high-speed modulation performance of QD lasers from a theoretical point of view, given some advances in tunnel injection as well as potential advances in dense nanoscale patterning to assess higher modulation speeds in the important 1.3- to $1.5-\mu \mathrm{m}$ window.

Also of interest is the use of QDs in new types of applications considered in an article by Botez et al. of the University of Wisconsin at Madison. Quantum cascade lasers (QCLs) have made much progress in recent times in the 3.6- to $15-\mu \mathrm{m}$ range but still lag far behind nearinfrared quantum-well diode lasers in wall-plug efficiency. QD-based lasers designed in a cascaded format using intersubband transitions may be a way to improve QCLs. Recent work on the subject is presented and discussed by Botez et al.

Next, the subject of QD photodetectors is concisely reviewed by Adrienne Stiff-Roberts of Duke University. The operation, epitaxial growth, and performance of standard InAs/GaAs quantum dot infrared photodetectors (QDIPs) are explicitly presented, suggesting that QDIPs are well-suited for detecting mid-infrared light at elevated temperatures and to reduce the cost of infrared imaging systems by enabling cryogenic dewars and Stirling cooling systems to be replaced by thermo-electric coolers. The QDIP performance is compared to commercially available technologies, and options for moving beyond InAs/GaAs QDIPs are discussed.

Distinct from the epitaxially grown QDs, advances in organometallic chemistry have given rise to the wet synthesis of colloidal semiconductor nanocrystal QDs. The solution-processed QDs can be made highly monodisperse and exhibit size-tunable bandgaps with nearly unitary florescence quantum yield, extremely narrow emission bandwidth, core-shell-enabled structural robustness, unmatched photochemical stability, and broad wavelength coverage over the entire visible spectrum $(400-700 \mathrm{~nm})$ and the technologically important near-infrared $(700-2500 \mathrm{~nm})$ regime. These superior properties render them promising building blocks for the next-generation thin-film optoelectronics, which has spurred the widespread interests of developing QD-based light emitting-diodes, hybrid photovoltaics, nanoscale lasers, etc. Toward this end, Menon et al. summarize their work on colloidal QD-based integrated photonic devices, including flexible microcavity lasers, microdisk emitters and integrated active-passive waveguides. In particular, the solution-processed microcavity laser structure can be removed off the substrate, thereby yielding a flexible structure that can conform to any shape and whose emission spectrum can be mechanically tuned. Furthermore, planar photonic devices consisting of vertically coupled microring resonators, microdisk emitters, activepassive integrated waveguide structures, and coupled active microdisk resonators have been realized using soft lithography, photo-lithography, and electron beam lithography, respectively. The gain medium in all these devices is a composite consisting of QDs embedded in a polymer matrix. The wet-synthesis method offers an inexpensive alternative to the traditional photonic device fabrication techniques.

Finally, utilizing the optical gain within and near-field optical coupling between the QDs, sub-diffraction optical energy transmission with low loss has been proposed and demonstrated by Lin et al. at the University of Washington, Seattle. Their QD waveguides utilize near-field optical coupling between QDs and nano-scale QD photodetectors with nanogaps for subdiffraction limit waveguiding and sensing with high spatial resolution and sensitivity, as well as integration of these two nanophotonic components. The QD waveguides achieve a transmission loss of $3 \mathrm{~dB} / 4 \mu \mathrm{m}$, which is lower than the experimental results from other subdiffraction-limit waveguides that have been reported to date. Lin et al. have also demonstrated a comparable waveguiding effect through a waveguide with a sharp bend. The QD photodetector shows a sensitivity of $60 \mathrm{pW}$ over a device with a nanogap of $25 \mathrm{~nm}$ for detection.

Just as we benefitted from organizing this special section, we hope that the readers of the journal will also. 